\title{
Azimuth Angle Distribution of the Secondary Particles of Cosmic-Ray Jets.
}

\author{
K. Imaeda and P. Fleming \\ School of Cosmic Physics, Dublin Institute for Advanced Studies - Dublin \\ (Nuovo Cimento, 62 A, $439(1969)$ )
}

i) In line 14 of page 441 : replace $\tilde{\beta}_{f}\left(=\tilde{\beta}_{f t}\right)$ by $\tilde{\beta}_{f}\left(=\gamma_{f} \beta_{f t}\right)$.

ii) Equation (3) should read

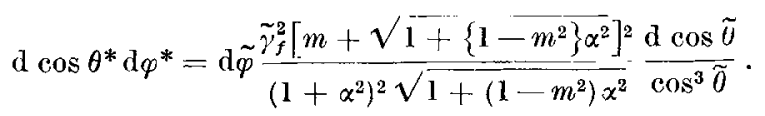

iii) Equation (5) should read

$$
\Phi(\phi) \mathrm{d} \phi=\frac{\tilde{\gamma}_{f}^{2}\left(1-m^{2}\right)^{\frac{3}{2}}}{2 \pi m^{2}} X^{2}\left[1+X\left(\frac{\pi}{2}+\operatorname{tg}^{-1} X\right)\right] \mathrm{d} \operatorname{tg} \phi,
$$

where

$$
X: \pm \sqrt{\frac{a^{2}}{1+\tilde{\gamma}_{f}^{2} \operatorname{tg}^{2} \phi}}, \quad a^{2} \equiv \frac{m^{2}}{1-m^{2}},
$$

iv) In equation (A.1), $x$ should read $x=\frac{\sqrt{2 \ln 2}}{\phi_{\frac{1}{2}}} \phi$.

v) In equation (A.2), the second term of the right-hand side $a_{0}$ should read $2 a_{0}$.

vi) Equation (A.3) should read

$$
N(0 \sim \xi)=\sqrt{2}\left(a_{0}+\pi k a_{0}^{2}\right) \xi \bar{k}+\frac{1}{\sqrt{2}}\left[\frac{1}{2}-G\left[\frac{k}{\sqrt{2}}(2 \pi-\xi)\right]+G\left[\frac{k \xi}{\sqrt{2}}\right]\right] .
$$

